\title{
Intelligent transportation systems in big data
}

\author{
Xiang $\mathrm{Li}^{1}$ \\ Published online: 11 September 2018 \\ c) Springer-Verlag GmbH Germany, part of Springer Nature 2018
}

\section{Introduction}

Big data provides unprecedented opportunities for the development of intelligent transportation systems. Taking full advantage of big data, we can accomplish informed decision-making and management, e.g., controlling risk, improving energy efficiency, reducing carbon emissions and others. The public transportation industry has been at the forefront in utilizing and implementing big data. Machine intelligence technologies have already been applied to this area.

This special issue aims to deliver a platform. On the platform, researchers coming from academe and industry can present the advanced methodologies to cope with intelligent transportation systems in Big Data problems. At the same time, they can also elaborate on the state-of-the-art case studies in selected areas of application through the usage of big data and reports on the linkages between methodology and practice of big data analysis.

Once the call for papers was announced in September 2017, this special issue has attracted tremendous attention. In total, we received 30 manuscripts. After a rigorous review process, 11 papers have been finally accepted for publication.

To gain a better insight into the essence of the special issue, we offer brief highlights of the contributing papers.

\section{Theory}

In order to enhance the performance of forecasting, the paper titled "A new information fusion method of forecasting" proposed a novel fusion method to make full use of all kinds of forecast information. The paper written by Y. Su and W. Sun. proposed a dynamic stochastic differential model for

Xiang Li

lixiang@mail.buct.edu.cn

1 School of Economics and Management, Beijing University of Chemical Technology, Beijing, China describing traffic flow based on the Markov chain theory. A key feature of the new approach is considering vehicle arrivals and departures, which results in the total number of vehicles in the system varying over time, which make the proposed transport system dynamic and more realistic. In the paper titled "Solving the green fuzzy vehicle routing problem using a revised hybrid intelligent algorithm", the fuel consumption and fuzzy travel time have been delineated in developing and solving the green-fuzzy vehicle routing problem as an extension of the celebrated VRP in which routes are performed to reduce the total expenditure. In view of that problem such as low scale merit, inefficient resource utilization and conflict of interest between the operator and customer in the process of network operation, the paper written by $\mathrm{Xu}$ et al. seeks to formulate the scheduling strategy for multiple logistics tasks oriented to multi-origin and multidestination on the basis of multiple-allocation-hybrid H\&S collaborative network. The paper written by $\mathrm{L}$. Wu et al. investigates the route choice problem on a network with uncertain travel times along the arcs and possible blocked nodes.

\section{Methodologies and case studies}

Chen et al. in the paper entitled "An entropy based solid transportation problem in uncertain environment" employs entropy function of dispersals of trips between origins and destinations as a second objective function so as to minimize the transportation penalties and ensure uniform distribution of goods between origins and destinations. The paper written by Li et al. analyzed the influence of traffic signs on the path selection behavior of pedestrians, and construct the MAKLINK diagram to study the impact of traffic signs on pedestrians' proceeding decisions by a simulation technique. B. Wang et al. in the paper entitled "Capacity optimization and allocation of an urban rail transit network based on multisource data" proposed a multi-objective optimization model for the capacity allocation of an urban rail transit network based on multi-source data on the Beijing metro passenger 
flow, which considers the operating costs of trains and the expenses related to the waiting time of transferring passenger. In order to alleviate the truck congestion in container terminals, issues of modeling truck congestion pricing are addressed in the paper titled "Bi-level programming model of truck congestion pricing at container terminals", in which a bi-level programming model is developed to determine the optimal toll rates. The paper written by Wang and Liu proposed the urban road network resilience evacuation method under snow event. In order to improve the resilience of road network, they established the mathematical model for road network recovery under extreme weather to solve the snow removal resource location-allocation problem (LAP) with uncertain weather information. A mixed transportation problem with centralized and decentralized transportation is proposed in collaborative logistics network. Deng et al. in the paper named "Route optimization model in collaborative logistics network for mixed transportation problem considered cost discount based on GATS" proposed a route optimization programming model to minimize the total costs in consideration of cost discount, road section capacity, overload penalty, advance delivery cost and hard time window.

We envision that the papers published in this special issue would be of interest to researchers and practitioners and help identify further research directions. We also hope that the readers can find the material of this special issue both informative and inspiring when exploring the field of optimization and decision-making.

Acknowledgements As Guest Editor, we would also like to thank all the referees for their support and professional service and express the gratitude to all authors for their submissions to this special issue. Without the support of the authors and the referees, it would have been impossible to make this special a reality.

Publisher's Note Springer Nature remains neutral with regard to jurisdictional claims in published maps and institutional affiliations. 